\title{
Neonatal Extremity Compartment Syndrome: A Rare Diagnosis Requiring Prompt Recognition
}

\author{
Nicholas T. Severyn, DO ${ }^{1}$ Kok Lim Kua, MD ${ }^{1}$ \\ ${ }^{1}$ Department of Pediatrics, Division of Neonatal-Perinatal Medicine, \\ Indiana University School of Medicine, Indianapolis, Indiana \\ Am J Perinatol Rep 2020;10:e386-e389.
}

\begin{abstract}
Address for correspondence Nicholas T. Severyn, DO, Department of Pediatrics, Neonatology Division, Indiana University School of Medicine, 1030 West Michigan Street, Suite C 4600, Indianapolis, IN 46202 (e-mail: nseveryn@iu.edu).
\end{abstract}

\author{
Abstract \\ Keywords \\ - compartment \\ syndrome \\ - fasciotomy \\ - neonatal
}

Neonatal extremity compartment syndrome is an extremely rare diagnosis. Risk factors that predispose infants to a hypercoagulable state or trauma have been implicated, but the exact mechanisms remain poorly understood. The hallmark of the condition is extremity swelling with sentinel skin changes. We report a case of upper extremity compartment syndrome from initial presentation until 3 months after discharge and discuss the importance of prompt diagnosis and timely surgical evaluation.
Upper extremity compartment syndrome leading to ischemia and contracture was first described by Volkmann in $1881 .{ }^{1}$ Neonatal compartment syndrome (NCS) is extremely rare, with less than 100 cases reported in the literature. ${ }^{2,3}$ Nearly all reported neonatal cases involved the upper extremities, and the affected limb presented with significant swelling and sentinel skin changes such as desquamation, blister/bullae formation, and skin necrosis. ${ }^{2-8}$ These skin findings are thought to reflect underlying tissue ischemia secondary to an increased interstitial pressure within a confined compartment. ${ }^{2,4}$ Risk factors that predispose infants to a hypercoagulable state (sepsis, thrombophilic disorders, maternal diabetes) or trauma (fetal macrosomia, difficult/traumatic birth extraction, oligohydramnios, amniotic band syndrome) have been implicated, but the exact cause of NCS remains poorly understood. ${ }^{2-5}$

The severity of NCS ranges from transient ischemia causing minimal damage to severe compartment syndrome causing growth restriction, severe contracture, loss of limb function, and limb necrosis. ${ }^{5}$ A previous case series and literature review of NCS reported at long-term follow-up $84 \%$ of infants had persistent contracture, $82 \%$ had radiographic bony abnormalities, and $45 \%$ had persistent nerve dysfunction. ${ }^{2,4}$ Multiple publications have showed that ear-

received

April 25, 2020

accepted after revision

August 21, 2020 lier surgical intervention is associated with better functional outcomes. $^{3-8}$ Therefore, it is important that clinicians caring for neonates recognize NCS and refer for surgical evaluation quickly. Given the rarity of the diagnosis, the association of symptoms is often not immediately appreciated, and emergent surgical intervention is rarely performed. ${ }^{2}$ We report the clinical progression of a late preterm infant with severe NCS that highlights the importance of quick recognition and surgical evaluation.

\section{Case Presentation}

A 29-year-old gravida 3 para 2 woman presented to a referral hospital with nonreassuring fetal heart tones at 33 3/7 weeks gestation. The pregnancy was complicated by insulin-dependent type 2 diabetes mellitus, hypertension, and obesity. Due to the nonreassuring fetal heart tones, the infant was delivered via urgent repeat cesarean section. Membranes were artificially ruptured at the time of delivery and fluid was meconium-stained. The infant was large for gestational age with a birth weight of $2,880 \mathrm{~g}$. Resuscitation was uncomplicated with Apgar scores of 8 and 8 at 1 and 5 minutes. The infant required $1 \mathrm{~L}$ nasal cannula at $60 \%$ oxygen for oxygen desaturations.
Copyright $\odot 2020$ by Thieme Medical Publishers, Inc., 333 Seventh Avenue, New York, NY 10001, USA. Tel: +1(212) 760-0888.
License terms $10.1055 / \mathrm{s}-0040-1721654$ ISSN 2157-6998.

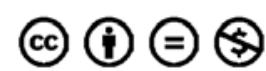




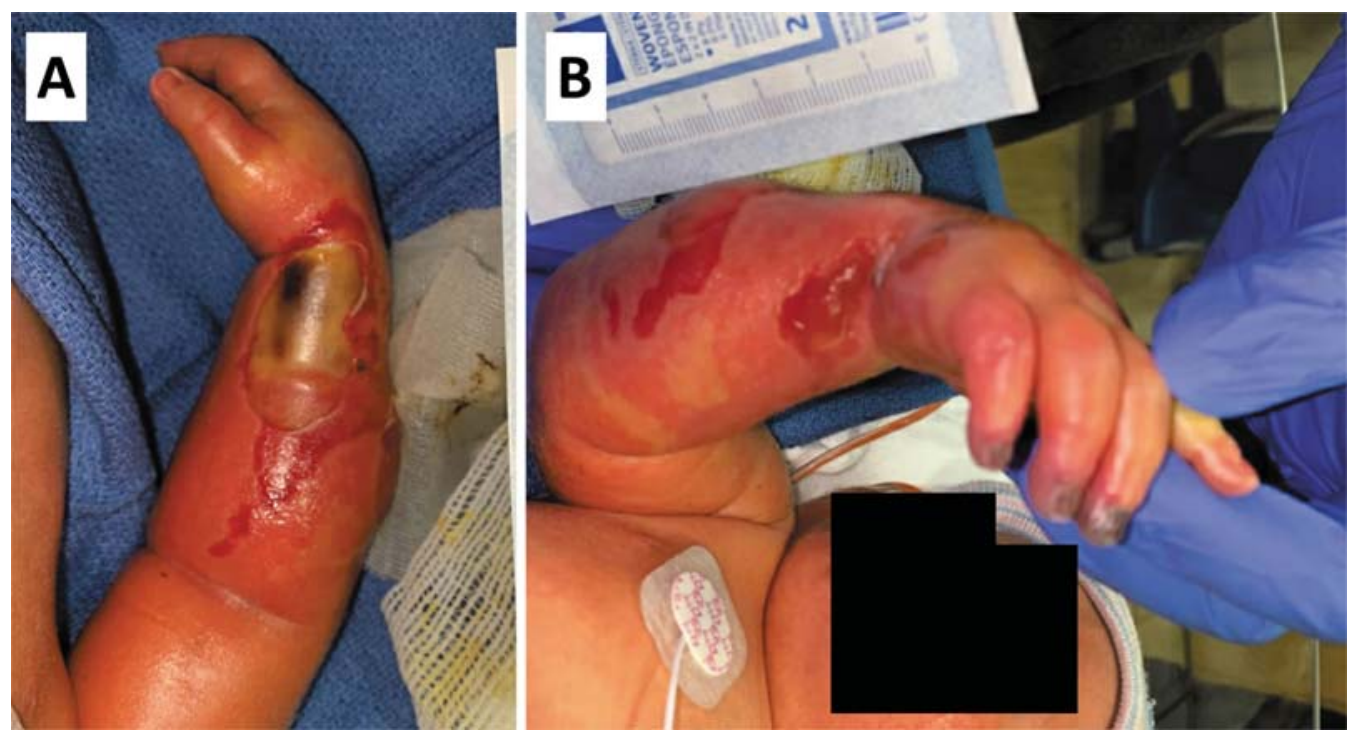

Fig. 1 (A) Right forearm and (B) right forearm and right hand at level IV neonatal intensive care unit.

At birth, the infant was found to have extensive swelling over the right forearm and right hand with multiple blistering skin lesions. Over the next 24 hours, the right forearm and hand swelling continued to worsen and developed decreased perfusion of the right distal digits that prompted the transfer to a level IV neonatal intensive care unit for further evaluation and possible surgical intervention.

On examination upon arrival at 38 hours of life, the infant's right forearm and right hand were edematous with very taut overlying skin. There were multiple ruptured blisters with the largest measuring $3.5 \mathrm{~cm}$ by $2 \mathrm{~cm}$ ( - Fig. 1A). The distal tips of the third, fourth, and fifth digits of the right hand were dusky with poor perfusion ( - Fig. 1B). Right brachial and radial arterial pulses were not palpable. Doppler exam detected soft pulsatile blood flow in the right brachial and radial arteries, but ulnar arterial blood flow was undetectable. The remainder of the infant's exam was unremarkable.

Herpes simplex virus polymerase chain reaction (HSV PCR) testing was performed from a swab of one of the lesions. Blood cultures were obtained and broad-spectrum antibiotics were started. Complete blood count and coagulation studies including prothrombin time, international normalized ratio, activated partial thromboplastin time, thrombin clotting time, and fibrinogen were unremarkable. Creatine kinase was elevated at 6,709 units/L. Plain films showed soft tissue swelling without any evidence of a fracture. A venous ultrasound was obtained that showed no evidence of deep vein thrombosis. Pediatric general surgery, vascular surgery, and plastic surgery were consulted. Based on the clinical findings, the diagnosis of compartment syndrome of the right forearm was made. The infant was taken emergently to the operating room for right forearm fasciotomies and carpal tunnel release 4 hours after arrival at 42 hours of life (-Fig. 2).

Postoperatively, right brachial, radial, and ulnar blood flow were all detected via Doppler. A brain magnetic resonance imaging/magnetic resonance angiography/magnetic

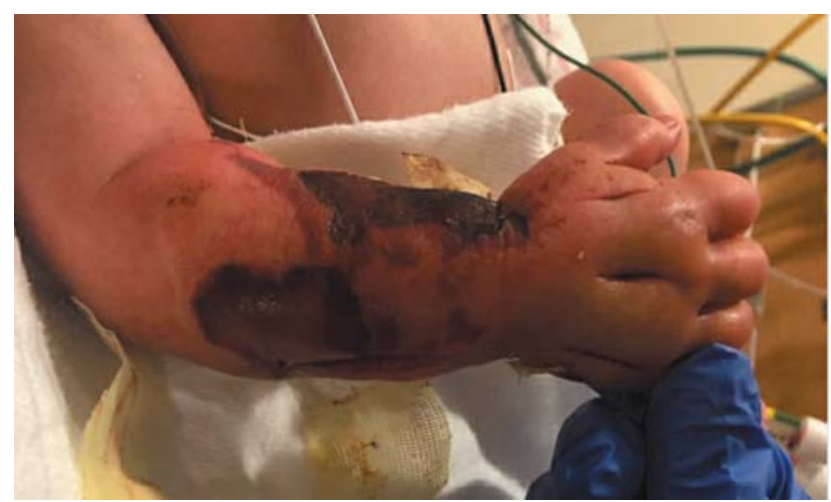

Fig. 2 Right forearm and hand after fasciotomies and carpal tunnel release.

resonance (MRI/MRA/MRV) was obtained that showed left grade II intraventricular hemorrhage, a small infarction in the right corpus striatum without hemorrhagic conversion, and normal vasculature. Neurology was consulted and felt the findings were compatible with prematurity. HSV PCR testing and blood cultures remained negative. Systemic antibiotics were continued while the fasciotomies were open.

The postoperative course was complicated by necrosis of the overlying skin, the distal forearm muscles, and the distal tips of the third, fourth, and fifth digits for which the infant required tissue debridement with allograft placement on days 5 and 14 of life. During the second debridement, the infant underwent amputation of the distal third, fourth, and fifth digits (-Fig. 3). On day of life 20, the infant underwent debridement again and a dermal matrix was placed in preparation of skin autografting. On day of life 43 , a skin autograft was placed and the infant was discharged the following day with close outpatient follow-up with plastic surgery. At 3-month follow-up, the infant continued to have a flexion deformity of the right wrist and fingers causing a limitation of passive extension to a neutral position, which was managed conservatively with a splint (- Fig. 4). 


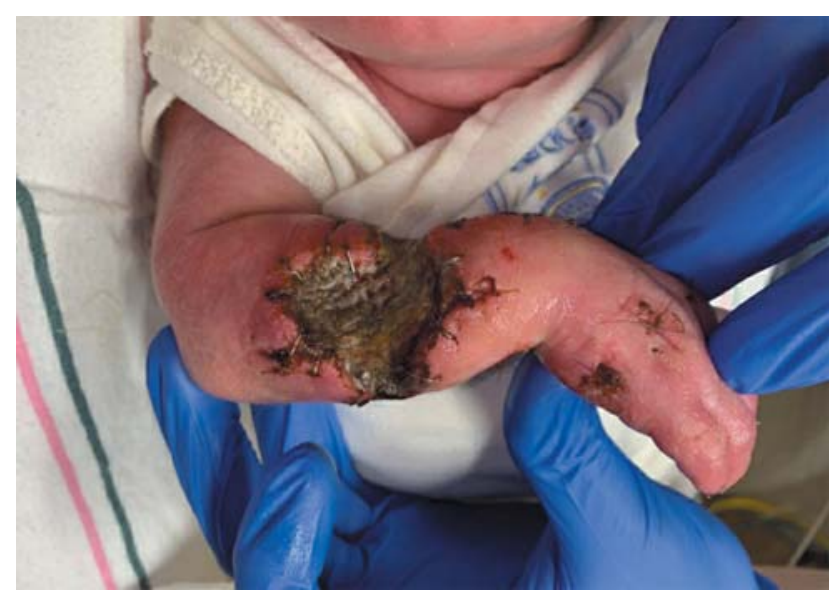

Fig. 3 Right forearm after second debridement and allograft placement and distal third, fourth, and fifth digit amputations.

\section{Discussion}

NCS remains a rare and poorly understood condition. Proposed intrinsic risk factors such as sepsis, thrombophilic conditions, and maternal diabetes predispose infants to a hypercoagulable state, potentially leading to an arterial or venous thrombus. ${ }^{2,4}$ In this case, anticoagulation was not initiated as there were no thrombus identified. Agrawal et al showed that empirical anticoagulation with no thrombus provided minimal clinical improvement. ${ }^{2}$ Given that a thrombus was not identified in the initial workup, we did not perform workup for hypercoagulability. On the other hand, infants with extrinsic risk factors such as oligohydramnios, prematurity, fetal distress manifesting as nonreassuring fetal heart tones, large fetal size for gestational age, traumatic birth extraction, in utero/peripartum limb compression injury, and amniotic band syndrome are more likely to develop localized tissue injury that could lead to swelling. In fact, over 20 neonatal conditions and comorbidities have been correlated with the development of compartment syndrome, but none have been directly implicated nor has any commonality been seen between cases. ${ }^{2,5}$ Given multiple intrinsic and extrinsic factors have been implicated, we propose two distinct mechanisms leading to the development of NCS. The first mechanism is driven by a hypercoagulable state that causes thromboembolic events, leading to tissue ischemia, reperfusion injury, and swelling. ${ }^{9,10}$ The second mechanism is initiated by trauma to the limb that causes skin changes and progressively worsening swelling. Ultimately, the common endpoint of these mechanisms involves severe swelling that impedes blood supply and causes further skin and tissue necrosis of the affected limb. Multiple risk factors were present in our case including fetal distress manifesting as nonreassuring fetal heart tones, large fetal size for gestational age, maternal diabetes, and prematurity. Details about labor, specifically details regarding the difficulty of extraction and limb malpositioning, were not reported. Based on the clinical presentation, we propose that the development of NCS in our patient was incited by an initial trauma to the affected upper extremity presenting with local skin findings with worsening swelling.

NCS is also associated with cerebral stroke. ${ }^{11}$ This association was attributed to a hypercoagulable state, and Pavlidis et al recommended neuroimaging to be performed on all newborns with extremity compartment syndrome. Indeed, MRI/MRA/MRV detected a small ischemic infarct in the right corpus striatum of our infant.

Although the exact etiology remains unknown, the presentation of NCS is very consistent. The majority of infants with NCS presented with swelling and skin changes over the
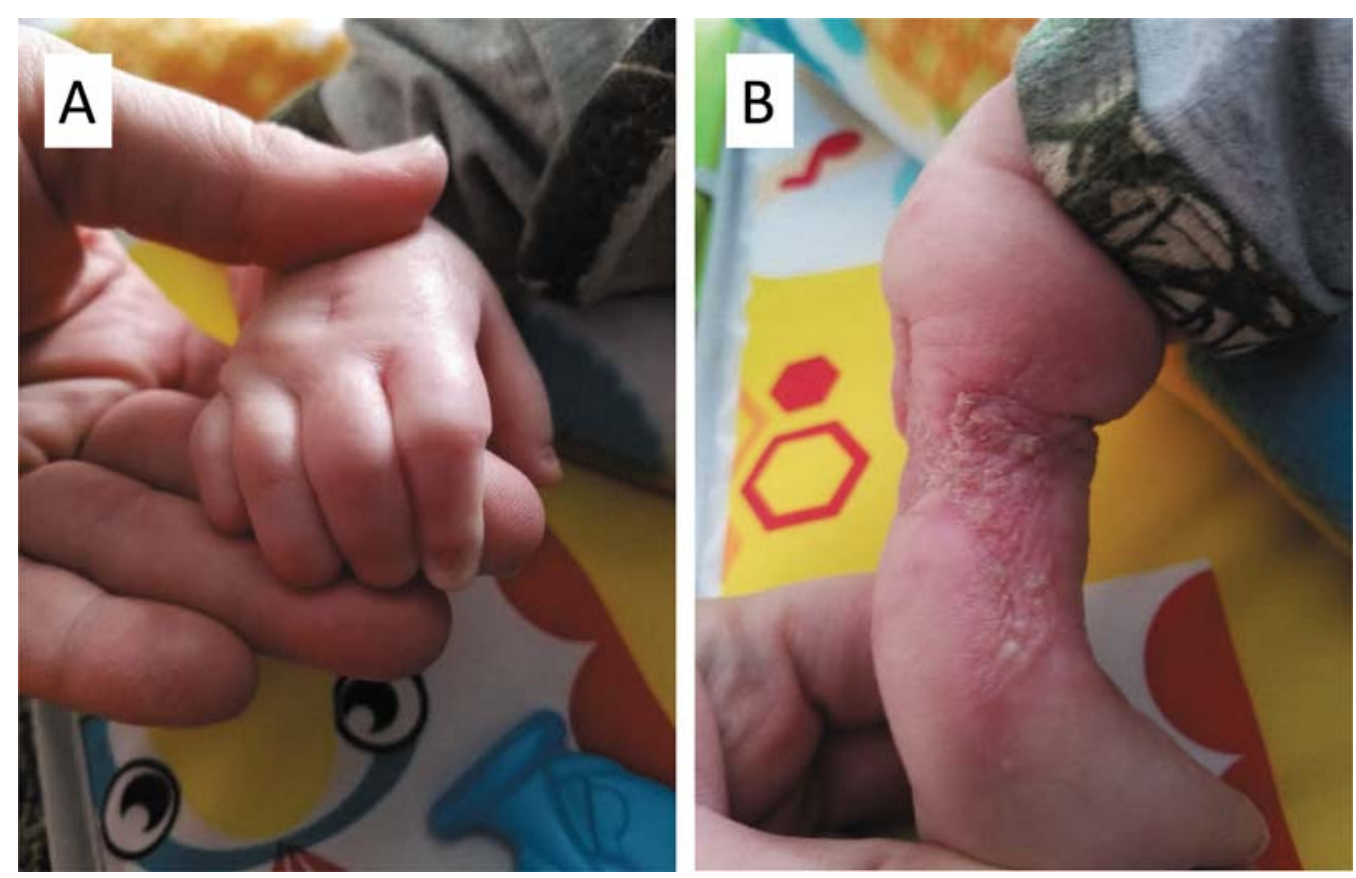

Fig. 4 (A) Right hand and (B) right forearm roughly 3 months after the initial fasciotomies. 
affected limb, which includes desquamation, blister/bullae formation, and skin necrosis. ${ }^{2-8}$ Despite these skin changes are well described as signs of severe compartment syndrome and raise the concern of extremely poor limb perfusion, fasciotomy was not emergently performed in many cases. ${ }^{2,4}$ In a review by Agrawal et al, $62 \%$ of infants had surgical intervention at some point including debridement and late reconstructive procedures, but only $14 \%$ underwent fasciotomy and $38 \%$ did not have any surgical intervention performed at all. ${ }^{2}$ The case series from Ragland et al echoed these findings, with $63 \%$ of their infants undergoing surgical intervention including debridement and reconstructive at some point, but only $8 \%$ underwent fasciotomy. ${ }^{4}$ The delay in diagnosis and surgery was associated with the need for multiple-staged surgical repairs, including debridement of necrotic tissue, reconstructive procedures, and in some cases amputation. ${ }^{2-4,8}$ While the evidence is scarce, these authors suggested that early fasciotomy, even when distal perfusion seems adequate, may be limb saving and lead to the best possible functional outcome..$^{2-4,8}$ In our case, the patient arrived at 38 hours of life and the diagnosis was made promptly, allowing emergent fasciotomy to begin at 42 hours of life. Despite this, our patient required multiple debridement and reconstructive procedures of the affected limb and continued to have a fixed-flexion deformity requiring splinting. While the prompt surgical intervention may have prevented limb loss in this case, it is uncertain if sooner intervention would have reduced the need for multiple procedures and decreased the risk of contracture development. The fact that the infant was not transferred until after 24 hours of life added further uncertainty as to whether a more timely surgical intervention would have resulted in a better outcome. As noted by multiple authors, the diagnosis of NCS is often delayed, likely secondary to the rarity of the condition leading to a delay in recognition of the syndrome by providers. ${ }^{2-4,8}$ This case report, along with the clinical images, will remind to clinician to recognize the classic presentation of this rare disorder. Given earlier surgical intervention likely gives the highest change of achieving the best function outcome, we recommend all infants with suspected NCS to be transferred as soon as possible to a center where prompt surgical evaluation can take place regardless if limb perfusion is not compromised.

\section{Conclusion}

NCS remains a rare condition of unknown etiology, with both intrinsic and extrinsic factors likely playing a role. Previous case reports suggest prompt diagnosis and timely surgical intervention lead to the best functional outcome, and our case of NCS adds evidence to this strategy. Newborn infants with suspected NCS, regardless if limb perfusion seems adequate, should be managed at a center with access to multiple surgical subspecialties, especially if multiple staged reconstructive procedures are anticipated.

\section{Financial Disclosure}

All authors have no financial relationships relevant to this article to disclose.

\section{Contributor's Statement Page}

Dr. Severyn obtained consent from the mother to present the case and use images.

Dr. Severyn and Dr. Kua conceptualized and designed the study, drafted the initial manuscript, and critically reviewed and revised the manuscript.

Both Dr. Severyn and Dr. Kua approved the final manuscript as submitted and agreed to be accountable for all aspects of the work.

\section{Funding Source}

No funding was secured for this study.

Conflict of Interest

All authors have no conflicts of interest to disclose.

\section{References}

1 Volkmann R. Die ischaemischen muskellahmungen and kontrakturen. Zentralbl Chir 1881;8:801-803

2 Agrawal H, Dokania G, Wu SY. Neonatal Volkmann ischemic contracture: case report and review of literature. AJP Rep 2014; 4(02):e77-e80

3 Allen LM, Benacci JC, Trane RN III, Driscoll RJ. A case of neonatal compartment syndrome: importance of early diagnosis in a rare and debilitating condition. Am J Perinatol 2010;27(02):103-106

4 Ragland R III, Moukoko D, Ezaki M, Carter PR, Mills J. Forearm compartment syndrome in the newborn: report of 24 cases. J Hand Surg Am 2005;30(05):997-1003

5 Martin B, Treharne L. Neonatal compartment syndrome. Ann R Coll Surg Engl 2016;98(07):e111-e113

6 Cham PM, Drolet BA, Segura AD, Esterly NB. Congenital Volkmann ischaemic contracture: a case report and review. $\mathrm{Br}$ J Dermatol 2004;150(02):357-363

7 Kline SC, Moore JR. Neonatal compartment syndrome. J Hand Surg Am 1992;17(02):256-259

8 Tetreault AK, Axibal DP, Scott FA. Neonatal compartment syndrome treated within the first 24 hours of life. Orthopedics 2018; 41(05):e731-e733

9 Bekmez S, Beken S, Mermerkaya MU, Ozkan M, Okumus N. Acute forearm compartment syndrome in a newborn caused by reperfusion after spontaneous axillary artery thrombosis. J Pediatr Orthop B 2015;24(06):552-555

10 Zajac JC, Mantilla-Rivas E, Tilt A, Bryant JR, Oh AK, Rogers GF. Neonatal compartment syndrome as a result of thromboembolic event. Plast Reconstr Surg Glob Open 2019;7(11):e2514

11 Pavlidis E, Spagnoli C, Duca M, Ormitti F, Magnani C, Pisani F. Neonatal forearm compartment syndrome: look for cerebral stroke. J Pediatr 2014;164(02):427.e1 